\title{
Quantitative computed tomography: emphysema and airway wall thickness by sex, age and smoking
}

\author{
T.B. Grydeland***, A. Dirksen ${ }^{\curvearrowleft}$, H.O. Coxson ${ }^{+}$, S.G. Pillai $^{\S}$, S. Sharma ${ }^{\S}$, G.E. Eide $^{f * *}$, \\ A. Gulsvik*,\# and P.S. Bakke*,\#
}

ABSTRACT: We investigated how quantitative high-resolution computed tomography (HRCT) measures of emphysema and airway wall thickness (AWT) vary with sex, age and smoking history.

We included 463 chronic obstructive pulmonary disease (COPD) cases and 431 controls. All included subjects were current or ex-smokers aged $\geqslant 40 \mathrm{yrs}$, and all underwent spirometry and HRCT examination. The HRCT images were quantitatively assessed, providing indices on lung density and airway dimensions.

The median (25-75th percentile) \%LAA950 (\% low-attenuation area < -950 HU) was 8.9 (3-19) and $4.7(1-16)$ in male and female COPD cases, respectively, and $0.71(0.3-1.6)$ and $0.32(0.1-0.8)$ in male and female controls, respectively. \%LAA950 was higher in ex-smokers and increased with increasing age and with increasing number of pack-years. The mean \pm SD standardised AWT was $0.504 \pm 0.030$ and $0.474 \pm 0.031$ in male and female COPD cases, respectively, and $0.488 \pm 0.028$ and $0.463 \pm 0.025$ in male and female controls, respectively. AWT decreased with increasing age in cases, and increased with the degree of current smoking in all subjects.

We found significant differences in quantitative HRCT measures of emphysema and AWT between varying sex, age and smoking groups of both control and COPD subjects.

KEYWORDS: Airway, chronic obstructive pulmonary disease, computed tomography, emphysema, sex, smoking

hronic obstructive pulmonary disease (COPD) is a complex disorder in which numerous parameters interact to produce chronic airflow limitation [1]. There are differences in the extent and expression of COPD between individuals, and it is thought that genetics play a key role in the development of the disease [2]. These differences are exemplified in the differential response to cigarette smoke seen in males and females [3, 4]. However, most studies investigating the extent of COPD and the differences between sexes are based on lung function tests and respiratory symptoms [5, 6] which, unfortunately, do not provide a complete picture of COPD.

Quantitative computed tomography (CT) has become a popular technique to quantify the changes in lung structure, and to separate different subtypes of COPD according to the contribution of airways disease and emphysema $[2,7,8]$. Other studies have examined the sex effects on the anatomical changes and have found that females have less emphysema at all levels of airflow obstruction $[3,9]$. However, there is very limited data available on how CT derived airway wall thickness (AWT) varies with age, sex and smoking history $[10,11]$. Such knowledge may work to increase our understanding of the natural course of COPD in males and females.

The objective of the present study was to quantify the emphysema and AWT in a large patient and community-based sample of ever-smokers, and to determine how these anatomical variables interact with sex, age and smoking to produce the clinical disorder known as COPD.

\section{METHODS}

This single centre study was conducted between January 2003 and January 2005 in Bergen, Norway. All subjects gave informed consent, and the local ethics committee and review board (Bergen) approved the study. The subjects included in the current study had all participated in the GenKOLS (Genetic COPD) study [12, 13], and constituted approximately half of the GenKOLS population (894 out of 1,909 subjects) who received an optional high-resolution CT (HRCT) scan. Subjects were recruited from a
AFFILIATIONS

*Dept of Thoracic Medicine, Haukeland University Hospital, ${ }^{f}$ Centre for Clinical Research, Haukeland University Hospital,

\#Institute of Medicine, University of Bergen, and

${ }^{* *}$ Dept of Public Health and Primary Care, University of Bergen, Bergen, Norway.

-Pulmonary Dept, Gentofte Hospital, Copenhagen, Denmark.

${ }^{+}$Dept of Radiology and James Hogg iCAPTURE Centre for Cardiovascular and Pulmonary Research, University of British Columbia, Vancouver, BC, Canada.

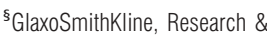
Development, Research Triangle Park, NC, USA.

CORRESPONDENCE

T.B. Grydeland

Dept of Thoracic Medicine

Haukeland University Hospital

$\mathrm{N}-5021$ Bergen

Norway

E-mail: thomas.grydeland@

med.uib.no

Received:

Nov 052008

Accepted after revision:

Feb 262009

First published online:

March 262009

European Respiratory Journa Print ISSN 0903-1936

Online ISSN 1399-3003 
hospital patient register and from two general population studies [14, 15], and a total of 463 COPD cases and 431 controls were included. Enrolment criteria were: 1) self-reported Caucasian; 2) aged $>40$ yrs; 3 ) current or former smoker with $\geqslant 2.5$ pack-yrs of smoking history; and 4) no severe $\alpha_{1}$ antitrypsin deficiency. Asthmatics were not excluded. For a complete list of inclusion and exclusion criteria refer to the supplementary data.

Subjects performed spirometry according to the American Thoracic Society standards [16], using a Vitalograph 2160 Spirometer (Vitalograph Ltd, Maids Moreton, UK) pre- and post-bronchodilation with $400 \mu \mathrm{g}$ salbutamol. Subjects were assessed at least 6 weeks after any respiratory infection, but were not asked to withhold regular medication. Local reference values for forced expiratory volume in $1 \mathrm{~s}$ (FEV1) and forced expiratory vital capacity (FVC) were used [17]. COPD cases had post-bronchodilator FEV1/FVC of $<0.70$ and an FEV1 $<80 \%$ predicted [1]. Controls had post-bronchodilator FEV1/FVC of $>0.70$ and an FEV1 $>80 \%$ pred.

\section{Computed tomography}

The HRCT scans were all performed using a GE LightSpeed Ultra CT scanner $(120 \mathrm{kVp}, 200 \mathrm{~mA}$; GE Healthcare, Milwaukee, WI, USA), at suspended full inspiration (apex to base) using 1-mm slice thickness at $20-\mathrm{mm}$ intervals, producing an average of $13.4 \pm 1.6$ slices per subject. The CT scans were reconstructed using both a low spatial frequency reconstruction algorithm (standard) for density measurements, and a high spatial frequency algorithm (bone) for airway measurements. All images were transferred to the James Hogg iCAPTURE Centre (Vancouver, BC, Canada) for quantitative analysis as previously described [18, 19]. Briefly, the lungs were segmented from the chest wall and central blood vessels and airways using a modified boarder tracing algorithm with prior position knowledge [20]. CT derived lung volume was also obtained. To obtain a proxy for inflation level, the CT derived total lung volume was divided by the predicted total lung capacity [21]. The extent of emphysema was assessed using the percentage of lung voxels with X-ray attenuation values less than (low attenuation areas; \%LAA) -950 Hounsfield units (HU) (\%LAA950). The -950 HU cut-off has been shown to be appropriate for this CT acquisition technique [22], but the amount of emphysema was also calculated using cut-offs of $910 \mathrm{HU}$ and $-856 \mathrm{HU}$ (data not shown). Airways cut in crosssection (short to long axis greater than 2:3) were identified on the CT scans and measured using the Full Width At Half Maximum algorithm [8]. To reduce technical errors associated with very small airways, only airways with an internal perimeter $>6 \mathrm{~mm}$ were included. To avoid potential bias issues surrounding different distribution of airway sizes between subjects, a standardised measure for AWT was derived for each subject by plotting the square root of the airway wall area against the internal perimeter of each measured airway (fig. 1). The resulting regression line was used to calculate the square root of the wall area for a 'theoretical airway' with an internal perimeter of $10 \mathrm{~mm}$ (AWT-Pi10) [2].

\section{Statistical analysis}

Proportions were tested using Chi-squared tests, while the mean was compared using ANOVA and the median using the
Kruskal-Wallis test. The associations of $\ln (\% \mathrm{LAA})$ and AWTPi10 to the explanatory variables were examined with multiple linear regression. In these analyses, the \%LAA values were transformed using the natural logarithm, because both the $\%$ LAA values and the regression residuals were right skewed. The following interactions were tested separately, based on an a priori assessment: sex $\times$ age, sex $\times$ pack-yrs and age $\times$ current smoking versus \%LAA, and sex $\times$ age and sex $\times$ current smoking versus AWT-Pi10. We also tested the COPD case-controlstatus $\times$ pack-yrs interaction versus both \%LAA and AWT-Pi10 on the whole material. A significance level of 0.05 was applied. All analyses were performed with STATA 9 (StataCorp LP, College Station, TX, USA).

\section{RESULTS}

For both sexes, COPD cases were older than controls, more COPD cases were current smokers and their total number of pack-years was higher than the controls (table 1). The number of pack-years in both COPD cases and controls was higher in males than in females, while the prevalence of current smokers was higher in females than in males. Male and female COPD cases were equally distributed across the Global Initiative for Chronic Obstructive Lung Disease (GOLD) stages; 60\% classified as stage II, $28 \%$ as stage III and the rest as stage IV.

The median \%LAA950 scores were higher in COPD cases and higher in males (table 2). AWT-Pi10 was higher in COPD cases and higher in males, regardless of disease status (table 2). Both lumen area and number of airways analysed was slightly higher in males, but there were no significant differences between COPD cases and controls.

The \%LAA950 increased with increasing age in both COPD cases and controls (table 2). The AWT-Pi10 decreased slightly with increasing age in COPD cases, but did not vary by age in controls. \%LAA950 was higher in ex-smokers than in current smokers. It also increased slightly with total smoking consumption in terms of pack-years, but did not vary by current number of cigarettes smoked daily. This pattern was seen regardless of disease status. The AWT-Pi10 increased slightly with higher daily cigarette consumption and increasing number of pack-years in both COPD cases and controls (table 2).

In the multiple linear regression analysis including sex, age and smoking, the relationships of sex and age to emphysema score and to the indices of AWT persisted (table 3). After adjusting for sex, age and daily cigarette consumption the \%LAA950 score increased with number of pack-years in COPD cases, while the AWT-Pi10 increased with number of packyears in controls. This adjusted analysis (table 3) also showed that the \%LAA950 decreased with increasing daily number of cigarettes smoked in COPD cases. AWT-Pi10 did not vary significantly with current daily number of cigarettes smoked.

The number of airways examined in each subject was also added to the airway models without affecting the overall results. Body mass index, post-bronchodilator FEV1 \% predicted, CT-measured total lung volume and total lung volume as a fraction of total lung capacity predicted (proxy for inflation level) were added separately to the models of table 3 . The explained variation was markedly increased, but the 


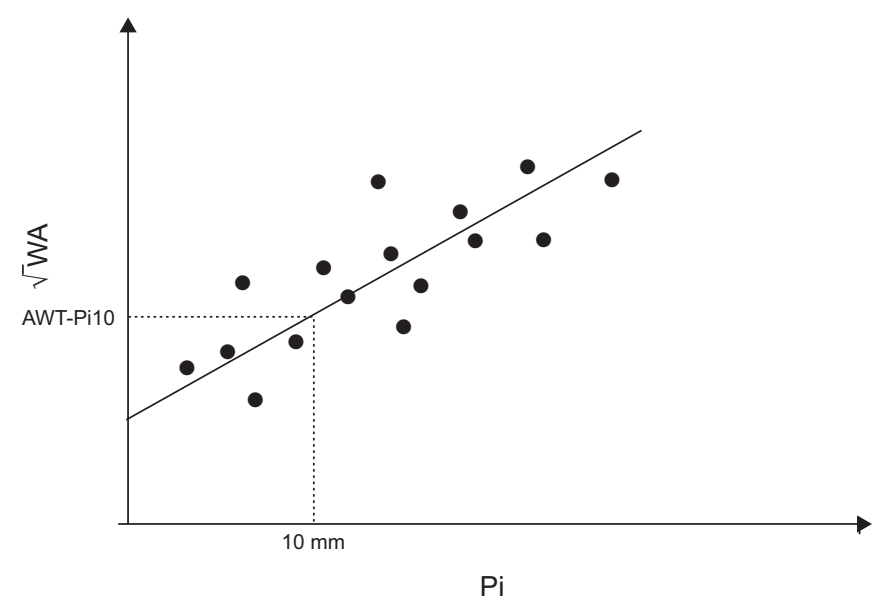

FIGURE 1. The calculation of AWT-Pi10. The square root of the wall area of each measured airway $(\sqrt{W} A)$ was plotted against the internal perimeter $(\mathrm{Pi})$ of that airway. This was performed separately for each subject. From the resulting regression line, a standardised measure of airway wall thickness for an airway with an internal perimeter of $10 \mathrm{~mm}$ was predicted (AWT-Pi10).

observed relationships remained overtly unchanged in size and direction.

We also calculated \%LAA910 and \%LAA856 (data not shown). They were analysed using the same multiple linear regression models shown in table 3, showing the same overall relationships found using \%LAA950.

\section{Interaction analysis}

The a priori defined interaction terms were then added separately to the multiple regression models. Sex and age showed a significant interaction in their effect on \%LAA950 among the controls, indicating that \%LAA950 increased more rapidly with increasing age in females than in males (fig. 2). Age and current smoking groups showed a significant interaction in their effect on \%LAA950 among COPD cases, indicating that \%LAA950 increased more rapidly with increasing age in current smokers (fig. 3). Sex and pack-years did not interact in their relationship to emphysema. Nor did sex and current smoking habit interact in their relationship to AWT.

We also combined the COPD cases and controls and added case/control status as an explanatory variable to the models in table 3. Case/control status and pack-years showed a significant interaction in their effect on AWT-Pi10, indicating that the AWT increased more rapidly with increasing number of pack-years in controls than in COPD cases (fig. 4).

\section{DISCUSSION}

In the current study we used a large COPD case-control population to perform sub-group analyses of the quantitative CT data and found considerable sex, age and smoking related differences in the estimates of emphysema and AWT. These data show that there is more emphysema in males and exsmokers and that the extent of emphysema increased with increasing age and number of pack-years, regardless of COPD case/control status. AWT was higher in males, and decreased slightly with increasing age in both COPD cases and controls.

\begin{tabular}{|c|c|c|c|c|}
\hline & \multicolumn{2}{|c|}{ COPD cases } & \multicolumn{2}{|c|}{ Control subjects } \\
\hline & Males & Females & Males & Females \\
\hline Subjects $n$ & 299 & 164 & 231 & 200 \\
\hline \multicolumn{5}{|l|}{ Demographics } \\
\hline Age yrs & $65.2 \pm 9.4$ & $62.6 \pm 9.0$ & $55.7 \pm 9.5$ & $54.1 \pm 8.9$ \\
\hline Ex-smokers & 53.2 & 46.3 & 62.3 & 52.5 \\
\hline Current smokers & 46.8 & 53.7 & 37.7 & 47.5 \\
\hline$<10$ cigarettes $\cdot$ day $^{-1}$ & 10.4 & 12.2 & 7.8 & 16.5 \\
\hline $10-19$ cigarettes $\cdot$ day $^{-1}$ & 21.4 & 30.5 & 21.2 & 23.0 \\
\hline$\geqslant 20$ cigarettes $\cdot$ day $^{-1}$ & 15.1 & 11.0 & 8.7 & 8.0 \\
\hline Pack-years & $30.6(21-43)$ & $22.5(16-32)$ & $18.0(10-27)$ & $14.0(8-23)$ \\
\hline Height cm & $175.3 \pm 6.8$ & $163.0 \pm 5.9$ & $178.2 \pm 6.6$ & $166.0 \pm 5.8$ \\
\hline Pre-BD FEV $1 \%$ pred & $49.5 \pm 17.4$ & $49.9 \pm 16.4$ & $91.9 \pm 9.1$ & $93.0 \pm 8.9$ \\
\hline Post-BD FEV 1 \% pred & $52.6 \pm 17.4$ & $53.1 \pm 16.1$ & $94.5 \pm 8.8$ & $96.0 \pm 9.1$ \\
\hline \multicolumn{5}{|l|}{ GOLD classification } \\
\hline Stage II & 58.2 & 62.8 & & \\
\hline Stage III & 28.1 & 28.1 & & \\
\hline Stage IV & 13.7 & 9.2 & & \\
\hline
\end{tabular}

Data are presented as mean \pm SD, per cent or median (25th-75th percentiles), unless otherwise stated. COPD: chronic obstructive pulmonary disease; BD: bronchodilator; FEV1: forced expiratory volume in $1 \mathrm{~s}$; \% pred: \% predicted; GOLD: Global Initiative for Chronic Obstructive Lung Disease.

Interaction analysis showed that the emphysema score increased more with increasing age in females and in current smokers. It also showed that the AWT increased more with increasing number of pack-years in the control group.

\section{Sex differences}

The data from this study confirm previous findings that there are more cases of emphysema in males than in females $[3,9]$ and expands the previous results by including control subjects with no airflow obstruction. This sex difference is contrary to the findings of GevenOIs et al. [23], who did not find any sex difference in CT lung densitometry; however, that study may have been underpowered by only including 42 subjects.

The higher emphysema score in males could have several explanations. Emphysema quantification is very sensitive to the level of inspiration, but even though males have larger lungs [17], there are no indications that males inspired deeper, and we did adjust the analyses for inspirational level. Environmental cause is another possible explanation, as males are more exposed to occupational airborne agents, which are potentially harmful to the lungs [24]. When our study group was in the first decade of life, the male smoking prevalence was two to three times that of females. As sons spend more time with their fathers than daughters do, they were more likely to be exposed to passive smoking [25]. There are data to suggest that passive smoking in childhood may have independent effects on the lungs in adult age [26]. Our findings could also be explained by residual confounding from smoking, but we were not able to examine the sex difference in never-smokers only, as these were not included in the study. 
TABLE 2 Quantitative high-resolution computed tomography parameters by sex, age and smoking groups

\begin{tabular}{|c|c|c|c|c|}
\hline & \multicolumn{2}{|c|}{$\%$ Low-attenuation areas $<-950 \mathrm{HU}$} & \multicolumn{2}{|c|}{ AWT-Pi10 cm ${ }^{\#}$} \\
\hline & COPD cases & Controls & COPD cases & Controls \\
\hline Subjects $n$ & 462 & 428 & 438 & 407 \\
\hline All subjects & $7.02(2.23-17.80)$ & $0.52(0.21-1.28)$ & $0.494 \pm 0.033$ & $0.477 \pm 0.02$ \\
\hline \multicolumn{5}{|l|}{ Sex } \\
\hline$p$-value & 0.0018 & 0.0001 & $<0.0001$ & $<0.0001$ \\
\hline \multicolumn{5}{|l|}{ Age yrs } \\
\hline$<50$ & 1.32 & 0.27 & 0.501 & 0.477 \\
\hline $50-59$ & 3.56 & 0.45 & 0.501 & 0.476 \\
\hline $60-69$ & 8.95 & 0.91 & 0.486 & 0.480 \\
\hline$\geqslant 70$ & 12.24 & 1.35 & 0.495 & 0.470 \\
\hline Current smokers & 4.86 & 0.35 & 0.495 & 0.476 \\
\hline $\mathrm{p}$-value & 0.0001 & 0.0001 & 0.4740 & 0.5683 \\
\hline \multicolumn{5}{|l|}{ Current smokers } \\
\hline$<10$ cigarettes $\cdot$ day $^{-1}$ & 6.02 & 0.31 & 0.480 & 0.473 \\
\hline 10-19.9 cigarettes $\cdot$ day $^{-1}$ & 4.28 & 0.36 & 0.499 & 0.473 \\
\hline$\geqslant 20$ cigarettes $\cdot$ day $^{-1}$ & 5.33 & 0.26 & 0.501 & 0.487 \\
\hline$p$-value & 0.1080 & 0.3414 & 0.0033 & 0.0493 \\
\hline \multicolumn{5}{|l|}{ Pack-years } \\
\hline$<15$ & 4.74 & 0.54 & 0.485 & 0.473 \\
\hline $15-29.9$ & 6.29 & 0.45 & 0.491 & 0.476 \\
\hline$\geqslant 30$ & 8.94 & 0.70 & 0.500 & 0.489 \\
\hline $\mathrm{p}$-value & 0.0733 & 0.3638 & 0.0033 & 0.0003 \\
\hline
\end{tabular}

However, we did compare subjects with $<10$ pack-yrs, and observed the same difference between males and females as in the entire sample (data not shown). Therefore, we find the residual confounding possibility less likely.

To our knowledge, we are the first to describe sex differences in CT measurements of airway dimensions. Our findings contradict the histological results in a subgroup of 101 subjects from the NETT study (the National Emphysema Treatment Trial), in which females had higher total wall area relative to luminal perimeter [3]. However, these studies are not comparable because the subjects in the NETT study were highly selected candidates for lung volume reduction surgery. Furthermore, the NETT subjects were all ex-smokers with no significant sputum production, while our study comprised a community-based sample of both current and ex-smokers with a wide range of respiratory symptoms and pulmonary function. Finally, the NETT data is based on airways $<2 \mathrm{~mm}$ in diameter measured using histology of the resected and most emphysematous part of the lungs, while our CT examination was based on airways $>2 \mathrm{~mm}$ in diameter from all regions of the lungs.
The sex difference in environmental exposures, described previously for the emphysema score, could also explain the airway differences. Furthermore, males have larger lungs than females [17], and that is the probable explanation of why we measure a slightly larger number of airways with slightly larger lumens in males. However, AWT-Pi10 adjusts for this difference and, after adding height or CT derived total lung volume to the model, the sex difference persisted. One could argue that the CT derived total lung volume is dependent on the degree of inspiration. Hence, we also adjusted for the CT derived total lung volume as a fraction of expected total lung capacity. Still the sex difference in AWT persisted, supporting the fact that this is a genuine sex difference, unrelated to the difference in size between sexes.

\section{Age differences}

The increasing emphysema score with ageing found in our study is in line with previous cross-sectional [23] and longitudinal [27] surveys. None of these studies examined if age and sex interacted in their effect on emphysema score, while we found that the sex difference in emphysema score 


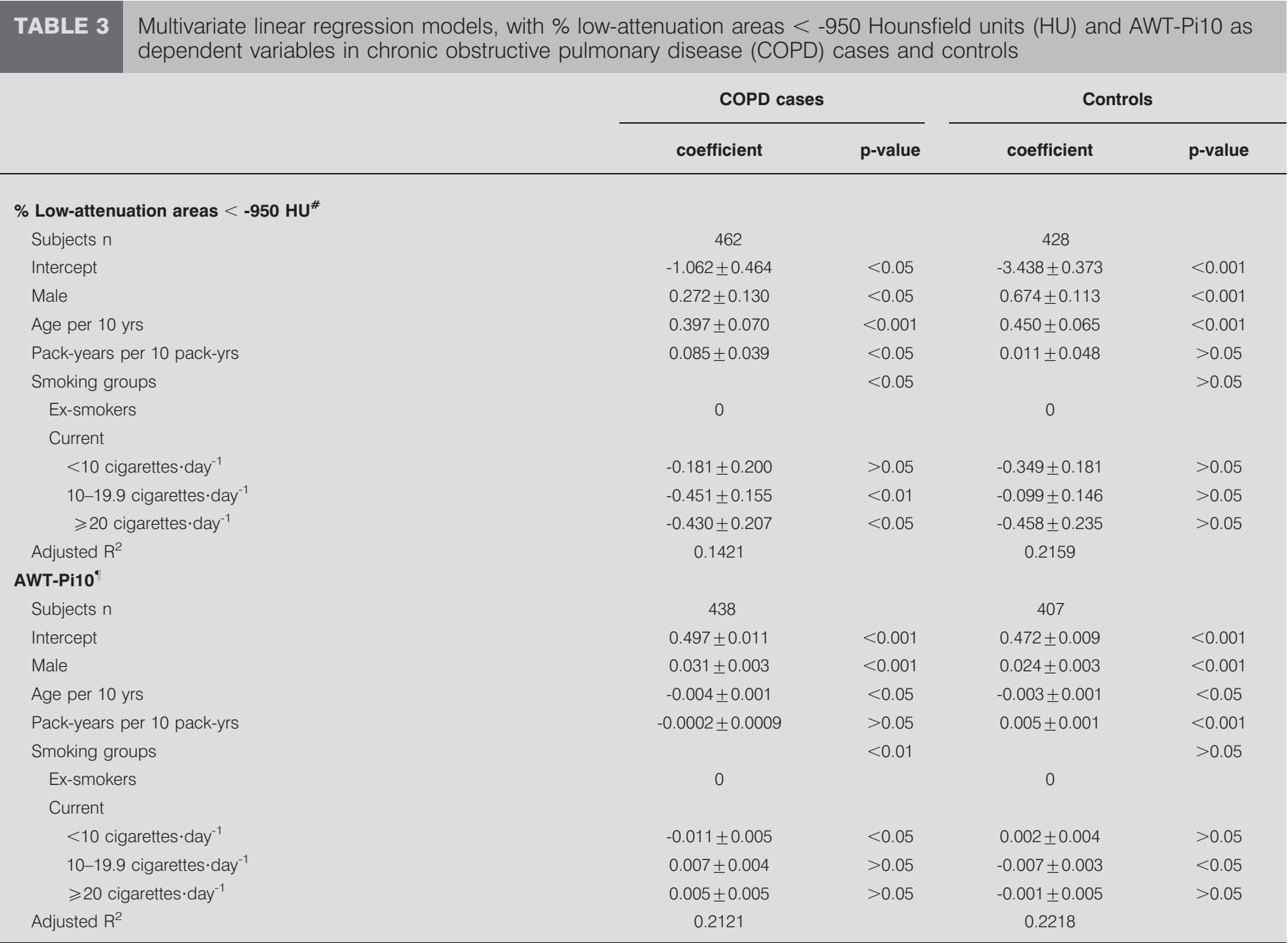

Data are presented as coefficient \pm SE, unless otherwise stated. AWT-Pi10: airway wall thickness for an airway with an internal perimeter of $10 \mathrm{~mm}$. ${ }^{\#}:$ log-transformed;

$\because$ : standardised measure of airway wall thickness.

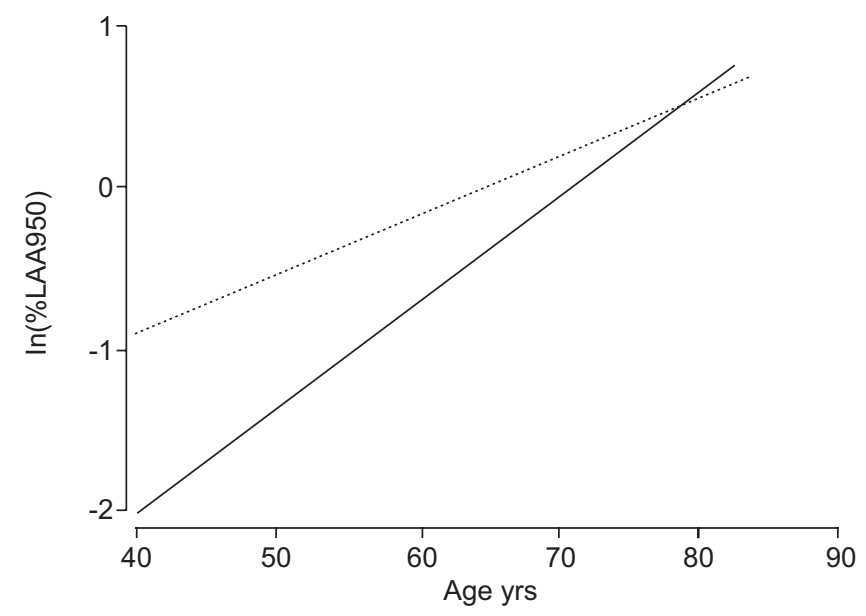

FIGURE 2. The relationship of log-transformed \% low-attenuation areas $<-950$ Hounsfield units (\%LAA950) and age in controls when pack-years and current smoking levels were held constant. There was a significant interaction between age and sex $(p=0.007)$. . .....: males; _ _ females. declined significantly with increasing age among the controls (fig. 2). This may have several explanations. It could indicate that the natural ageing of the lung gradually reduces the relative importance of environmental risk factors to which males are more exposed. It could also be a selection effect more pronounced in males than females as the age-sex interaction was only present in the control group. Finally, it could be related to the fact that the increased deposition of body fat associated with ageing is mainly abdominal in males, and more evenly distributed in females [28, 29]. This increased amount of abdominal fat in ageing males could, in the supine CT measurement position, press the diaphragm cranially, causing increased pulmonary density and reducing the percentage of tissue with a density $<-950 \mathrm{HU}$. In support of this, we also found that CT measured lung volume was slightly reduced in elderly male controls. It is also worth mentioning that age was a stronger predictor for emphysema than smoking was in both COPD cases and controls, and this is in line with data recently reported in the BOLD study (Burden of Chronic Obstructive Lung Disease) [30]. 


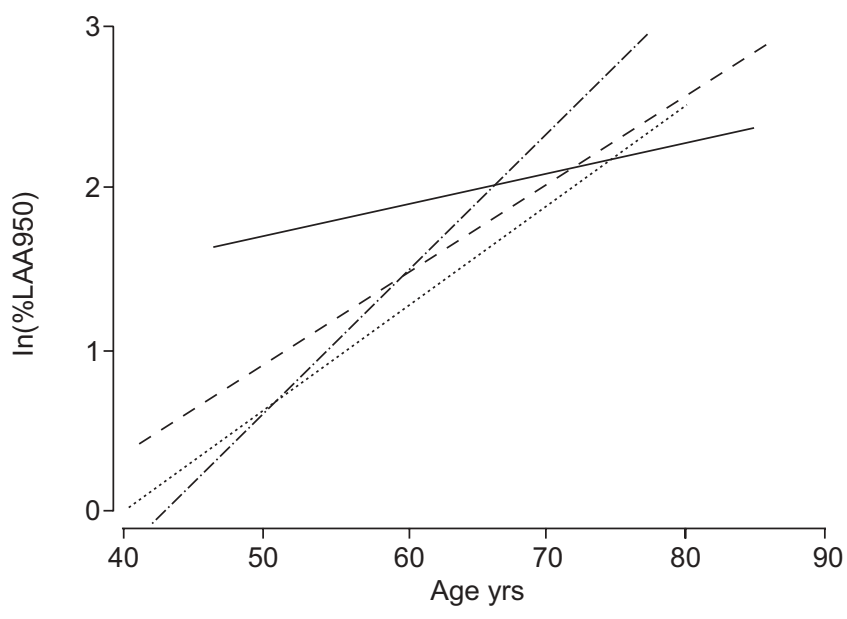

FIGURE 3. The relationship of log-transformed \% low-attenuation areas $<-950$ Hounsfield units (\%LAA950) and age in chronic obstructive pulmonary disease cases when pack-years and sex were held constant. There was a significant interaction between age and current smoking status $(p=0.006)$. ex-smoker; - - - - -: current smoker, $<10$ cigarettes $\cdot$ day $^{-1} ; \cdot \cdots \cdots$... current smoker, 10-20 cigarettes $\cdot$ day $^{-1} ; \cdot \cdot \cdot \cdot-\cdot$ current smoker, $\geqslant 20$ cigarettes $\cdot$ day $^{-1}$.

\section{Smoking-related differences}

Increasing number of pack-years smoked was associated with increased emphysema score in COPD cases, even after adjusting for sex, age and current smoking status (table 3). This is in accordance with the study by SoEjIMA et al. [27], which followed 83 smokers with annual CT for 5 yrs, even though they did not adjust for age. These data are also in line with previous respiratory physiological studies which report that pack-years are an independent predictor for indices of emphysema [31]. The association between emphysema and pack-years, as well as the greater increase of emphysema with increasing age in current smokers (fig. 3), was seen in our COPD cases only. However the controls had smoked, and were smoking significantly less and had significantly less emphysema than the COPD cases.

The decrease in \%LAA950 associated with increased current daily smoke consumption in COPD cases (tables 2 and 3) is most probably due to a survivor effect, where those with more advanced disease would be more likely to reduce or quit smoking.

No clear trend was seen for the quantity of current smoking. However, the number of pack-years was related to increasing AWT in controls (table 3; fig. 4). This could imply that the observed airway wall thickening, which on average was larger among COPD cases, is caused by different underlying pathophysiological mechanisms in COPD cases than controls [32-35]. However, our study is cross-sectional, and to clarify these relationships a longitudinal design is needed.

\section{Strengths and limitations of the study}

The strength of this study is that it is a single centre study, and all control subjects were sampled from a community study with high response rates [36-38]. Furthermore, surveys of nonresponders have shown that the study samples are representative of the population at large with respect to sex, age and smoking [36], and we think that the external validity of

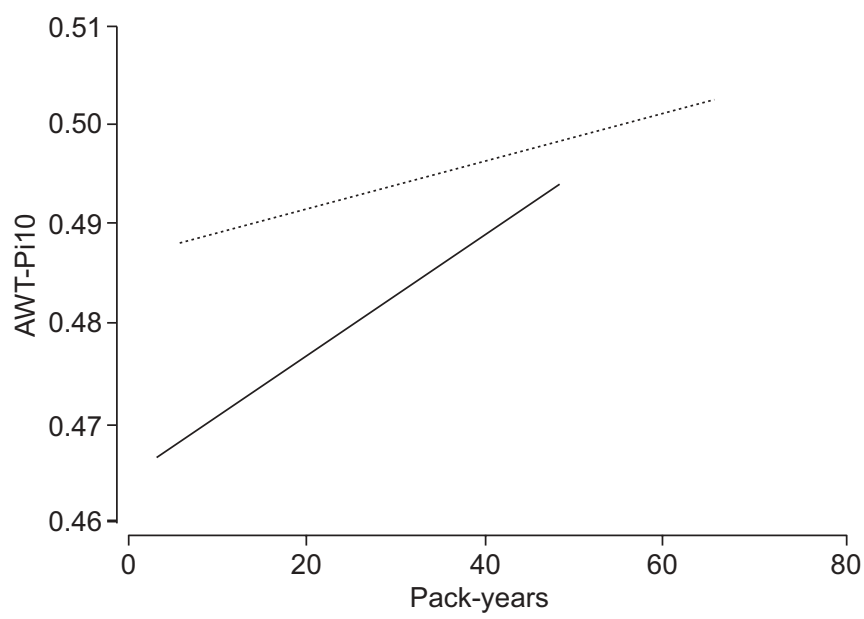

FIGURE 4. The relationship of AWT-Pi10 (standardised measure of airway wall thickness for an airway with an internal perimeter of $10 \mathrm{~mm}$ ) and pack-years when age, current smoking level and sex were held constant. There is a significant interaction between pack-years and case-control status $(p=0.003) . \cdots \cdots \cdots \cdot$ : chronic obstructive pulmonary disease cases; ___ controls.

our findings is high. Finally, all CT scans were performed with the same scanner.

However, there are some limitations to this study. 1) Even though subjects with a total smoking history as low as 2.5 pack-yrs were included in the study, we did not include never-smokers. 2) There were more male COPD cases, and the COPD cases were older, but all these differences have been adjusted for in the multivariate analyses. 3) The study did not include GOLD stage I. However, GOLD stages II-IV are those regarded as clinically important [30]. 4) The CT scans were not spirometrically gated, but it has been shown that the repeatability of quantitative $\mathrm{CT}$ tests is high and not likely to improve by using spirometric gating [39]. Furthermore, all analyses were adjusted for inflation level. 5) We were not able to determine the generations of the airways measured, and the generations might have differed between those with small and large lungs. However, adjustments for lung volume, lumen size or number of airways measured did not alter the relationships observed in table 3. Finally, it has been shown that CT measurements of small airway wall dimensions using the technique employed here are extremely variable [40]. Therefore, we only used larger airways in our analysis. While it is well established that COPD is a small airways disease [41, 42], studies have shown that the inflammatory response seen in small airways can also be seen in large airways [43], and that CT estimates of AWT are correlated with histological measurements of small airway wall dimensions [8]. Therefore, we are confident that our results do reflect the changes that are occurring in the small airways.

In conclusion, this study shows that the sex, age and smoking composition of the study population has strong effects on the quantitative CT measures of emphysema and AWT, and must be considered when comparing CT studies. As long as these population characteristics are taken into account, quantitative $\mathrm{CT}$ is a valuable tool for further characterisation of not only COPD phenotypes, but also healthy subjects to understand the natural history of the lung. 


\section{SUPPORT STATEMENT}

This work was supported by GlaxoSmithKline.

\section{STATEMENT OF INTEREST}

Statements of interest for H.O. Coxson, S.G. Pillai and S. Sharma and the study itself can be found at www.erj.ersjournals.com/misc/ statements.dtl

\section{ACKNOWLEDGEMENTS}

We would like to thank E. Thorsen for valuable comments during the preparation of this manuscript, C. Storness-Bliss for quantitative analysis and A-T. Tran for development and maintenance of the analysis software and database.

\section{REFERENCES}

1 Rabe KF, Hurd S, Anzueto A, et al. Global strategy for the diagnosis, management, and prevention of chronic obstructive pulmonary disease: GOLD executive summary. Am J Respir Crit Care Med 2007; 176: 532-555.

2 Patel BD, Coxson HO, Pillai SG, et al. Airway wall thickening and emphysema show independent familial aggregation in chronic obstructive pulmonary disease. Am J Respir Crit Care Med 2008; 178: 500-505.

3 Martinez FJ, Curtis JL, Sciurba F, et al. Sex differences in severe pulmonary emphysema. Am J Respir Crit Care Med 2007; 176: 243-252.

4 Becklake MR, Kauffmann F. Gender differences in airway behaviour over the human life span. Thorax 1999; 54: 1119-1138.

5 Langhammer A, Johnsen R, Gulsvik A, et al. Forced spirometry reference values for Norwegian adults: the Bronchial Obstruction in Nord-Trøndelag Study. Eur Respir J 2001; 18: 770-779.

6 Holmen TL, Barrett-Connor E, Clausen J, et al. Gender differences in the impact of adolescent smoking on lung function and respiratory symptoms. the Nord-Trøndelag Health Study, Norway, 1995-1997. Respir Med 2002; 96: 796-804.

7 de Jong PA, Long FR, Wong JC, et al. Computed tomographic estimation of lung dimensions throughout the growth period. Eur Respir J 2006; 27: 261-267.

8 Nakano Y, Wong JC, de Jong PA, et al. The prediction of small airway dimensions using computed tomography. Am J Respir Crit Care Med 2005; 171: 142-146.

9 Dransfield MT, Washko GR, Foreman MG, et al. Gender differences in the severity of CT emphysema in COPD. Chest 2007; 132: 464-470.

10 Coxson HO. Quantitative computed tomography assessment of airway wall dimensions: current status and potential applications for phenotyping chronic obstructive pulmonary disease. Proc Am Thorac Soc 2008; 5: 940-945.

11 Dirksen A. Is CT a new research tool for COPD? Clin Respir J 2008; 2: Suppl. 1, 76-83.

12 Zhu G, Warren L, Aponte J, et al. The SERPINE2 gene is associated with chronic obstructive pulmonary disease in two large populations. Am J Respir Crit Care Med 2007; 176: 167-173.

13 Kauffmann F. SERPINE2 and COPD. Am J Respir Crit Care Med 2007; 176: 725-726.

14 Eagan TM, Gulsvik A, Eide GE, et al. Remission of respiratory symptoms by smoking and occupational exposure in a cohort study. Eur Respir J 2004; 23: 589-594.

15 Brogger J, Eagan T, Eide GE, et al. Bias in retrospective studies of trends in asthma incidence. Eur Respir J 2004; 23: 281-286.

16 Standards for the diagnosis and care of patients with chronic obstructive pulmonary disease. American Thoracic Society. Am J Respir Crit Care Med 1995; 152: S77-S121.

17 Gulsvik A, Tosteson T, Bakke P, et al. Expiratory and inspiratory forced vital capacity and one-second forced volume in asymptomatic never-smokers in Norway. Clin Physiol 2001; 21: 648-660.
18 Rogers RM, Coxson HO, Sciurba FC, et al. Preoperative severity of emphysema predictive of improvement after lung volume reduction surgery: use of CT morphometry. Chest 2000; 118: 1240-1247.

19 Coxson $\mathrm{HO}$, Rogers RM, Whittall KP, et al. A quantification of the lung surface area in emphysema using computed tomography. Am J Respir Crit Care Med 1999; 159: 851-856.

20 Coxson HO, Mayo JR, Behzad H, et al. Measurement of lung expansion with computed tomography and comparison with quantitative histology. J Appl Physiol 1995; 79: 1525-1530.

21 Miller MR, Crapo R, Hankinson J, et al. General considerations for lung function testing. Eur Respir J 2005; 26: 153-161.

22 Gevenois PA, de Maertelaer V, De Vuyst P, et al. Comparison of computed density and macroscopic morphometry in pulmonary emphysema. Am J Respir Crit Care Med 1995; 152: 653-657.

23 Gevenois PA, Scillia P, de Maertelaer V, et al. The effects of age, sex, lung size, and hyperinflation on CT lung densitometry. AJR Am J Roentgenol 1996; 167: 1169-1173.

24 Bakke PS, Hanoa R, Gulsvik A. Relation of occupational exposure to respiratory symptoms and asthma in a general population sample: self-reported versus interview-based exposure data. Am J Epidemiol 2001; 154: 477-483.

25 US Department of Health and Human Services. The Health Consequences of Involuntary Exposure to Tobacco Smoke: a Report of the Surgeon General. Atlanta, GA, U.S. Department of Health and Human Services, Centers for Disease Control and Prevention, Coordinating Center for Health Promotion, National Center for Chronic Disease Prevention and Health Promotion, Office on Smoking and Health, 2006. www.surgeongeneral.gov/ library/secondhandsmoke/report/

26 Skorge TD, Eagan TM, Eide GE, et al. The adult incidence of asthma and respiratory symptoms by passive smoking in uterus or in childhood. Am J Respir Crit Care Med 2005; 172: 61-66.

27 Soejima K, Yamaguchi K, Kohda E, et al. Longitudinal follow-up study of smoking-induced lung density changes by high-resolution computed tomography. Am J Respir Crit Care Med 2000; 161: 1264-1273.

28 Cotes JE, Chinn DJ, Reed JW. Body mass, fat percentage, and fat free mass as reference variables for lung function: effects on terms for age and sex. Thorax 2001; 56: 839-844.

29 Chinn DJ, Cotes JE, Reed JW. Longitudinal effects of change in body mass on measurements of ventilatory capacity. Thorax 1996; 51: 699-704.

30 Buist AS, McBurnie MA, Vollmer WM, et al. International variation in the prevalence of COPD (the BOLD study): a population-based prevalence study. Lancet 2007; 370: 741-750.

31 Welle I, Eide GE, Bakke P, et al. Applicability of the single-breath carbon monoxide diffusing capacity in a Norwegian community study. Am J Respir Crit Care Med 1998; 158: 1745-1750.

32 James AL, Wenzel S. Clinical relevance of airway remodelling in airway diseases. Eur Respir J 2007; 30: 134-155.

33 McParland BE, Macklem PT, Pare PD. Airway wall remodeling: friend or foe? J Appl Physiol 2003; 95: 426-434.

34 Saetta M, Turato G, Baraldo S, et al. Goblet cell hyperplasia and epithelial inflammation in peripheral airways of smokers with both symptoms of chronic bronchitis and chronic airflow limitation. Am J Respir Crit Care Med 2000; 161: 1016-1021.

35 Van Pelt W, Quanjer PH, Borsboom GJ, et al. Respiratory symptoms and the maximum expiratory flow-volume curve; a multivariate approach. Eur Respir J 1988; 1: 122-132.

36 Eagan TM, Eide GE, Gulsvik A, et al. Nonresponse in a community cohort study: predictors and consequences for exposure-disease associations. J Clin Epidemiol 2002; 55: 775-781.

37 Bakke P, Gulsvik A, Lilleng P, et al. Postal survey on airborne occupational exposure and respiratory disorders in Norway: causes and consequences of non-response. J Epidemiol Community Health 1990; 44: 316-320. 
38 Brogger J, Bakke P, Eide GE, et al. Contribution of follow-up of nonresponders to prevalence and risk estimates: a Norwegian respiratory health survey. Am J Epidemiol 2003; 157: 558-566.

39 Gierada DS, Yusen RD, Pilgram TK, et al. Repeatability of quantitative CT indexes of emphysema in patients evaluated for lung volume reduction surgery. Radiology 2001; 220: 448-454.

40 Nakano Y, Whittall KP, Kalloger SE, et al. Development and validation of human airway analysis algorithm using multidetector row CT. Proc SPIE 2002; 4683: 460-469.
41 Hogg JC, Chu F, Utokaparch S, et al. The nature of small-airway obstruction in chronic obstructive pulmonary disease. $N$ Engl J Med 2004; 350: 2645-2653.

42 Hogg JC. Pathophysiology of airflow limitation in chronic obstructive pulmonary disease. Lancet 2004; 364: 709-721.

43 Tiddens HA, Pare PD, Hogg JC, et al. Cartilaginous airway dimensions and airflow obstruction in human lungs. Am J Respir Crit Care Med 1995; 152: 260-266. 